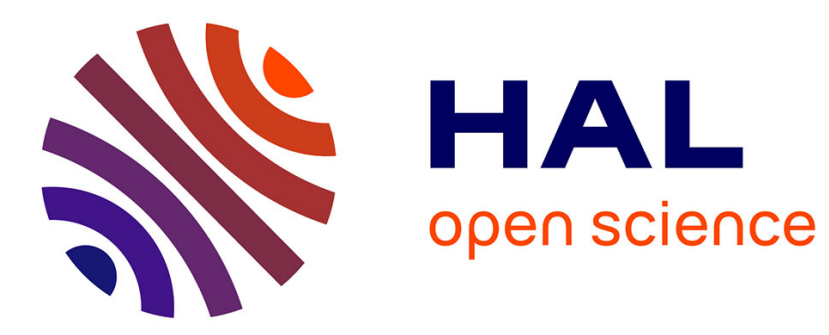

\title{
Diffraction par un corps élancé en mouvement
}

\author{
M. Tran van Nhieu
}

\section{To cite this version:}

M. Tran van Nhieu. Diffraction par un corps élancé en mouvement. Journal de Physique IV Proceedings, 1994, 04 (C5), pp.C5-881-C5-884. 10.1051/jp4:19945192 . jpa-00252875

\section{HAL Id: jpa-00252875 https://hal.science/jpa-00252875}

Submitted on 1 Jan 1994

HAL is a multi-disciplinary open access archive for the deposit and dissemination of scientific research documents, whether they are published or not. The documents may come from teaching and research institutions in France or abroad, or from public or private research centers.
L'archive ouverte pluridisciplinaire HAL, est destinée au dépôt et à la diffusion de documents scientifiques de niveau recherche, publiés ou non, émanant des établissements d'enseignement et de recherche français ou étrangers, des laboratoires publics ou privés. 


\title{
Diffraction par un corps élancé en mouvement
}

\author{
M. TRAN VAN NHIEU
}

Thomson Sintra Uwa, 1 Avenue Aristide Briand, 94117 Arcueil, France

Application of the so-called slender-body approximation to calculate the field scattered by a uniformly moving slender-body of revolution insonified by a co-moving point source in free space. By using a linear transformation of coordinates analogous to a Lorentz transformation, the transformed problem is similar the stationary case except for the boundary condition on the body surface which is modified by the source and target motions. An approximate solution to the velocity potential is derived by applying the matched asymptotic expansions method. From a geometrical analysis of the previous results, a general solution is proposed for low subsonic motions at low frequency.

\section{Introduction}

La diffraction acoustique par des corps en mouvement est un problème difficile et les solutions analytiques sont peu nombreuses même pour des corps dits "canoniques". La méthode utilisée en Electromagnétique consiste à résoudre le problème dans un référentiel lié à la cible pour se ramener ainsi au cas stationnaire; la solution trouvêe est ensuite réexprimée en fonction des variables du référentiel de départ [1]. Nous allons appliquer cette méthode pour déterminer le potentiel des vitesses diffracté par un corps élancé de révolution supposé infiniment rigide insoné par une source ponctuelle omnidirectionnelle. Dans la première section, où la cible et la source d'émission sont en mouvement de translation uniforme, le problème est résolu à partir de la solution établie en régime stationnaire [2]; une solution approchée est ensuite proposée dans la deuxième section pour des mouvements quelconques de la source et de la cible aux faibles nombre de Mach à basses fréquences.

\section{Théorie}

Soit un référentiel fixe (F) lié au fluide ambiant supposé immobile, homogène et infini et un référentiel mobile (C) lié au corps; l'origine de (C) noté O est pris au centre de l'objet. On désigne par L sa demi-longueur et a le rayon du maitre-couple (voir Figure 1). Lorsque la cible et l'émetteur sont en mouvement rectiligne uniforme avec une même vitesse de déplacement $\mathrm{V}$, on peut construire une solution analytique à partir des résultats obtenus en régime stationnaire $(\mathrm{V}=0)$ par une transformation linéaire des variables spatio-temporelles. Afin de simplifier l'analyse théorique, on se limitera dans ce paragraphe à un corps de révolution en translation suivant l'axe FZ. La source émettrice $Q$ supposée ponctuelle est de la forme 


$$
Q=Q_{0} e^{-i \omega T} \delta\left(X-X_{s}\right) \delta\left(Y-Y_{s}\right) \delta\left(Z-Z_{s}\right)
$$

où (Xs,Ys,Zs) sont les coordonnées de la source et $Q_{0}$ son intensité .

On désigne par $\left(\mathrm{X}^{2}+\mathrm{Y}^{2}\right)^{1 / 2}=\mathrm{aF}[(\mathrm{Z}-\mathrm{VT}) / \mathrm{L}]$ l'équation de la surface du corps supposée infiniment rigide.

Soit le changement de variables $(\mathrm{x}, \mathrm{y}, \mathrm{z}, \mathrm{t})$ suivant qui laisse invariant l'opérateur d'Alembertien [3].

$$
x=\gamma X ; y=\gamma Y ; z=\gamma^{2}(Z-V T) ; t=\gamma^{2}\left(T-\frac{V}{c^{2}} Z\right) ; \gamma=1 / \sqrt{1-M^{2}}
$$
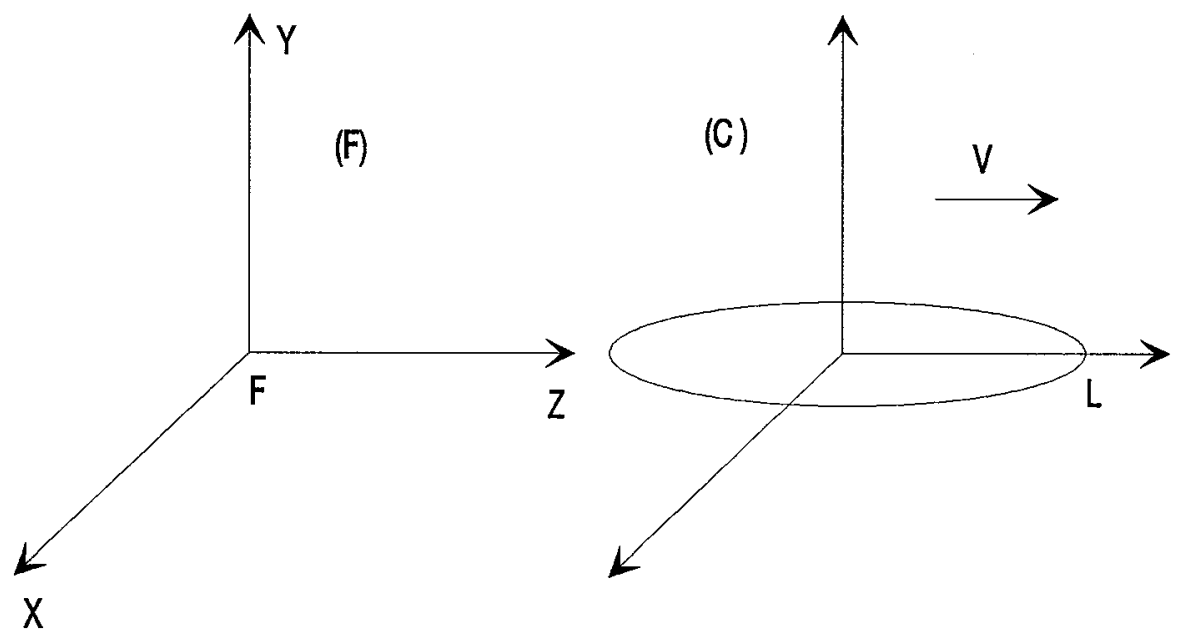

Figure 1: Géométrie du problème

En normalisant les longueurs par $L$, le temps par $L / c$ où $c$ désigne la célérité du son, le potentiel de vitesse par $Q_{0} L^{2}$, les équations du problème en variables adimensionnelles deviennent :

i) Pour l' équation de propagation

$$
\left(\Delta-\frac{\partial^{2}}{\partial t^{2}}\right) \psi=\gamma^{2} e^{-i k M z_{s}} e^{-i k t} \delta\left(x-x_{s}\right) \delta\left(y-y_{s}\right) \delta\left(z-z_{s}\right)
$$

où $\Psi$ désigne le potentiel des vitesses normalisé, $M=V / c$ le nombre de Mach et $k=\omega \mathrm{L} / \mathrm{c}$ le nombre d'onde acoustique divisé par $L$.

ii) Pour la condition à la surface du corps

$$
r=\epsilon \gamma F\left(z / \gamma^{2}\right): \frac{\partial \Psi}{\partial r}-\epsilon \gamma F^{\prime}\left(z / \gamma^{2}\right)\left(\frac{\partial \psi}{\partial z}-M \frac{\partial \Psi}{\partial t}\right)=0
$$

où la notation ' désigne la dérivée par rapport à l'argument tandis que $\varepsilon^{=} \mathrm{a} / \mathrm{L}$ est le paramètre d'élancement; pour les corps élancés $\varepsilon$ est très petit devant l'unité $\varepsilon \ll<1$.

Ces équations sont très semblable au cas stationnaire à l'exception de la présence de $\gamma$ et du 
facteur proportionnel à $\mathrm{M}$ dans l'équation (4). En posant

$$
\Psi(x, y, z, t, \epsilon, M)=\gamma^{2} e^{-i k t} e^{i M z_{s}} \Psi_{0}(x, y, z, \epsilon, M)
$$

et en ne retenant que le premier ordre en $\varepsilon, \Psi_{0}$ vérifie alors l'équation de Helmholtz mais avec une condition aux limites à la surface du corps modifiée par l'effet du mouvement. En procédant de la même manière que pour le cas stationnaire [2], la solution est décomposée en un champ incident $\Psi_{s}$ correspondant au potentiel des vitesses crée par le rayonnement d' une source omni-directionnelle en translation uniforme [3] et en un champ diffracté $\Psi \mathbf{r}$; on a

$$
\Psi_{0}=\Psi_{s}+\Psi_{r}
$$

Dans la présente analyse, la source est supposée localement plane le long de l'axe du corps dans le repère $(\mathrm{O}, \mathrm{x}, \mathrm{y}, \mathrm{z})$. Conformément à notre approche acoustique des corps élancés [4], le champ diffracté est recherché sous la forme d'une distribution continue d'harmoniques sphériques sectoriels en fonction des coordonnées $(x, y, z)$. Les calculs sont relativement longs mais restent similaire à ceux de la Réf.[2]; on peut montrer en appliquant l'approximation des corps élancés que

$$
\Psi_{r}=i \frac{\gamma^{4}}{\pi} \sum_{n=-\infty}^{\infty} e^{i n\left(\phi-\phi_{s}\right)}(-1)^{n} \int_{-1}^{1} a_{n}(s)\left(\frac{\sin \theta}{\sin \theta_{s}}\right)^{|n|} \frac{e^{-i k \Lambda}}{\rho \rho_{s}} d s
$$

où les intensités $a_{n}$ sont obtenus par raccordement avec la solution intérieure

$$
\begin{gathered}
a_{n}(s)=\frac{J_{n}^{\prime}-i \delta F^{\prime} J_{n}}{H_{n}^{1}-i \delta F^{\prime} H_{n}^{1}}\left[k \in \gamma F(s) \sin \theta_{s}\right] \\
\delta=\epsilon \gamma \frac{\left(\cos \theta_{s}+M\right)}{\sin \theta_{s}} ; \rho=\sqrt{\left(z-\gamma^{2} s\right)^{2}+r^{2}} ; \sin \theta=r / \rho ; r=\sqrt{x^{2}+y^{2}}
\end{gathered}
$$

Dans l'équation (7), on peut montrer que l'argument $\Lambda$ sous le terme intégral à une interprétation géométrique simple en fonction des retards de temps

$$
\Lambda=c\left(T-\tau-\tau_{s}\right)
$$

où $\tau$ est le temps que met le son pour aller d'un point d'observation du repère fixe (F) au point d'intégration $M$ situé sur l'axe du corps dans le repère mobile $(C)$; de même $\tau_{s}$ est le temps que met l'onde acoustique pour aller du point $M$ au point d'émission $S$.

Les équations (7)-(10) sont les principaux résultats de cet article; elles permettent de déterminer le champ acoustique diffracté en fonction des différents paramètres du problème; elles ont été déduites rigoureusement à partir d'un processus asymptotique et sont valable en basses fréquences pour $\omega \mathrm{a} / \mathrm{c}$ inférieur à l'unitê $\omega \mathrm{a} / \mathrm{c}=\mathrm{o}(1)$. On pourra noter que pour $\mathrm{M}<<1$, les termes intervenant dans ces équations sont proches du cas stationnaire à l'exception du terme de phase $\Lambda$ qui est rapidement oscillant. 
Cette remarque nous permet de généraliser les résultats précédents aux cas des mouvements quelconques lorsque les vitesses de déplacement sont petites devant la célérité du son.

\section{Approximation quasi-statique}

On considère une source $S$ et un corps élancé de révolution animés de mouvements arbitraires; dans le référentiel fixe (F), la trajectoire de $\mathrm{S}$ est caractérisée par les équations

$$
X_{s}=X_{s}(T) ; Y_{s}=Y_{s}(T) ; Z_{s}=Z_{s}(T)
$$

tandis que les coordonnées d'un point $M$ situé sur l'axe longitudinal du corps et d'abscisse $s$ dans le repère $(C)$ est défini par :

$$
X_{M}=X(s, T) ; Y_{M}=Y(s, T) ; Z_{M}=Z(s, T)
$$

L'approximation que nous proposons consiste à calculer le champ diffracté à partir de l'équation (7) mais en prenant:

. d'une part $M=0$ et $\gamma=1$ dans les équations (8) et $(9) ;(x, y, z)$ et $\left(x_{s}, y_{s}, z_{s}\right)$ étant les coordonnées instantanées du point d'observation et de $S$ dans le référentiel $(C)$ lié au corps.

. d'autre part calculer les retards $\tau$ et $\tau_{s}$ de l'équation (10) avec les deux équations suivantes:

$$
\begin{gathered}
c \tau=\sqrt{[X-X(s, T-\tau)]^{2}+[Y-Y(s, T-\tau)]^{2}+[Z-Z(s, T-\tau)]^{2}} \\
c \tau_{s}=\sqrt{\left[X_{s}\left(T-\tau_{s}\right)-X\right]^{2}+\left[Y_{s}\left(T-\tau_{s}\right)-Y\right]^{2}+\left[Z\left(T-\tau_{s}\right)-Z\right]^{2}}
\end{gathered}
$$

où $(\mathrm{X}, \mathrm{Y}, \mathrm{Z})$ sont les coordonnées du point d'observation dans le repère $(\mathrm{F})$.

On pourra vérifier que les rêsultats du précédent paragraphe sont obtenus en utilisant les expressions ad hoc pour les trajectoires de la source $\mathrm{S}$ et du corps. Enfin l'expression de la pression acoustique est obtenue par dérivation du potentiel des vitesses par rapport au temps.

\section{Conclusion}

Une expression du potentiel des vitesses diffracté par un corps élancé insoné par une source ponctuelle animés tous les deux d'un mouvement de translation uniforme est élaborée à partir d'une transformation linéaire des variables pour se ramener à un problème stationnaire; la solution du problème transformé est ensuite établie dans l'approximation des corps élancés, une interprétation géométrique simple permet d'étendre la solution pour des mouvements arbitraires de la cible et de la source d'émission. Cette solution est valable aux basses fréquences pour des longueurs d'onde grandes devant les dimensions transversales du corps et pour des vitesses de déplacemment petites devant la vitesse du son. Enfin les effets Doppler peuvent être déduits par dérivation du terme de phase de la solution obtenue.

\section{Références}

[1] Censor D., Radio Science 2 (1972) 331-337

[2] Tran Van Nhieu M., IEEE on UFFC 40 (1993) 325-329

[3] Morse P.M. and Ingard K.U. , Theoretical Acoustics (Mac Graw-Hill, 1968) pp. 718-723.

[4] Tran Van Nhieu M., J.Acoust.Soc.Am. 85 (1989) 1834-1840 